\title{
NEGATIVE FLOW OF ENERGY IN A MECHANICAL WAVE
}

\author{
A. Matulis ${ }^{a}$ and A. Acus ${ }^{b}$ \\ a Semiconductor Physics Institute, Center for Physical Sciences and Technology, Sauletekio 3, 10222 Vilnius, Lithuania \\ ${ }^{\mathrm{b}}$ Institute of Theoretical Physics and Astronomy, Vilnius University, Sauletekio 3, 10222 Vilnius, Lithuania \\ Email: matulisalg@gmail.com; arturas.acus@tfai.vu.lt
}

Received 30 October 2020; accepted 6 November 2020

\begin{abstract}
A classical system, which is analogous to the quantum one with a backflow of probability, is proposed. The system consists of a chain of masses interconnected by springs and attached by other springs to fixed supports. Thanks to the last springs the cutoff frequency and dispersion appears in the spectrum of waves propagating along the chain. It is shown that this dispersion contributes to the appearance of a backflow of energy. In the case of the interference of two waves, the magnitude of this backflow is an order of magnitude higher than the value of probability backflow in the mentioned quantum problem. The equation of Green's function is considered and it is shown that the backflow of energy is also possible when the system is excited by two consecutive short pulses. This classical backflow phenomenon is explained by the branching of energy flow to local modes that is confirmed by the results for the forced damped oscillator. It is shown that even in such a simple system the backflow of energy takes place (both instantaneous and average).
\end{abstract}

Keywords: wave, dispersion, energy, backflow, 1D lattice, oscillator

\section{Introduction}

There has always been an interest in essential quantum phenomena without classical analogues. The negative probability flow was believed to be such case, which was first mentioned by Allcock [1]] when he considered the time of arrival in quantum mechanics. Later this phenomenon was described in detail by Bracken and Melloy [2]. Exhaustive survey of quantum models and a broad review of the literature pertaining to this problem can be found in Yearsley's et al. paper [3] and Goussev's papers [4, 5]. The problem of negative flow was also considered in the field of optical phenomena [6]. There have been attempts to show that the effect of the negative flow may take place when replacing the quantum description with the classical one (see, for instance, Villanueva's paper [㠼). Gous- sev's paper [8] asserts that the effect of the negative probability flow occurs not only for a free quantum particle, but also for an ensemble of free classical particles with a Gaussian distribution of positions and momenta. This is quite expectable because the particle ensemble always shows a greater variety of motion types than the movement of an individual particle [9].

We believe that the phenomenon of the negative energy flow is inherent to any wave equation, whether it is related to the quantum model or to the classic one. The only important condition is the presence of dispersion that is most often due to local degrees of freedom in the media where the waves propagate. The aim of this paper is to demonstrate that the negative energy flow has to take place in a simple mechanical system: the chain of masses interconnected by harmonic springs, and 
its continuous version - the waves in an elastically braced string. In addition, we consider the finite version of the above chain in the limit case of a short length, thus turning into the problem of a harmonic oscillator.

The paper is organized as follows. In Section 2, a description of the basic model is provided, and in Section 3, the energy flows are introduced and the continuous limit of the model is formulated. In Section 4, the energy flow of the individual sinusoidal wave is calculated, and in Section 5, it is shown that in the case of two sinusoidal waves the local negative energy flow becomes possible. In Section 6, we consider the Green's function behaviour and show that the string demonstrates the local negative energy flow when it is excited by two consecutive pulses. In Section 7, we deal with a harmonic oscillator as the ultimate case of a supershort chain, which actually demonstrates the analog to the backflow of energy. Our conclusions are presented in Section 8.

\section{Model}

We consider the simple 1D (one dimensional) mechanical system shown in Fig. 1. It consists of

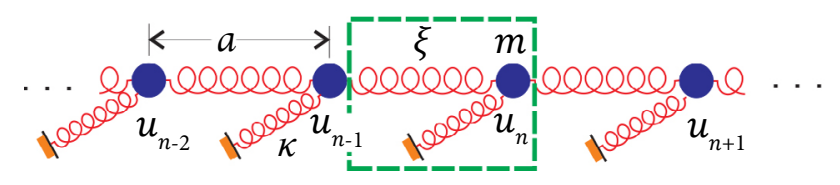

Fig. 1. Basic 1D lattice. Its primitive cell is shown by a green dashed rectangle.

point masses $m$ (indicated by filled circles) spaced the distance $a$ apart and interconnected by linear springs of stiffness $\xi$. Each mass is also attached to other spring of stiffness $\kappa$. The ends of these additional springs are kept at fixed positions. These extra springs provide us with the necessary wave dispersion. The masses are allowed to move in the horizontal direction only, and the symbols $u_{n}$ stand for their coordinates: the deviations from the equilibrium positions.

The dynamics of this $1 \mathrm{D}$ lattice is characterized by the following Hamiltonian:

$$
H=\frac{1}{2} \sum_{n=-\infty}^{\infty}\left[p_{n}^{2} / m+\kappa u_{n}^{2}+\xi\left(u_{n}-u_{n-1}\right)^{2}\right] .
$$

Here the symbol $p_{n}$ stands for the canonical momentum, conjugate to each coordinate $u_{n}$. The resulting equations of motion are

$$
\begin{aligned}
& \dot{u}_{n}=p_{n} / m, \\
& \dot{p}_{n}=m \ddot{u}_{n}=-\kappa u_{n}+\xi\left(u_{n-1}-2 u_{n}+u_{n+1}\right) .
\end{aligned}
$$

\section{Energy and its flow}

Energy and its flow appear naturally, calculating the first integral of differential motion equations. Indeed, multiplying Eq. (2b) by $\dot{u}_{n}$ we rewrite it as follows:

$$
\frac{\mathrm{d}}{\mathrm{d} t}\left(\frac{m}{2} \dot{u}_{n}^{2}+\frac{\kappa}{2} u_{n}^{2}\right)=\xi \dot{u}_{n}\left(u_{n-1}-2 u_{n}+u_{n+1}\right) .
$$

The expression in parentheses on the left-hand side of this equation looks like energy of the primitive cell. Consequently, its right-hand side has to be related to the energy flow. In order to make a final decision, however, it is necessary to take into account the potential energy of the string connecting neighbouring masses $\xi\left(u_{n}-u_{n-1}\right)^{2} / 2$ (see the Hamiltonian (1)). The above equation then turns into the following one:

$$
\begin{aligned}
\dot{\varepsilon}_{n} & =\frac{\mathrm{d}}{\mathrm{d} t}\left[\frac{m}{2} \dot{u}_{n}^{2}+\frac{\kappa}{2} u_{n}^{2}+\frac{\xi}{2}\left(u_{n}-u_{n-1}\right)^{2}\right] \\
& =\xi\left[\dot{u}_{n-1}\left(u_{n-1}-u_{n}\right)-\dot{u}_{n}\left(u_{n}-u_{n+1}\right)\right] .
\end{aligned}
$$

Actually it is the energy conservation rule applied to the primitive cell shown in Fig. 1 by a dashed rectangle ${ }^{1}$. It is obvious that the energy of the chosen primitive cell changes because a certain amount of energy flows into it, or flows out. These energy flows are actually determined by the expression on the right-hand side of Eq. (4). In this way, we will determine the flow of energy along the chain as the energy flowing through the right edge of the mentioned primitive cell (or through mass at $n$ )

$$
J_{n}=\xi \dot{u}_{n}\left(u_{n}-u_{n+1}\right)
$$

\footnotetext{
1 It is worth noting that the choice of a primitive cell is rather arbitrary, but it does not affect our further conclusions.
} 
that actually means the work done by mass $m$ in a unit of time over the spring attached to this mass on its right. In this case, the value of $J_{n-1}$ can be seen as the flow of energy entering the primitive cell on its left side, and Eq. (4) itself determines the energy balance of this primitive cell.

Due to the linearity of Eq. (2b), its solution can be presented as an exponential running wave

$$
u_{n}=\mathrm{e}^{\mathrm{i}(k a n-\omega t)},
$$

satisfying the dispersion relation

$$
\omega=\sqrt{4 \omega_{0}^{2} \sin ^{2}(k a / 2)+\Omega^{2}},
$$

where

$$
\Omega^{2}=\kappa / m \text { and } \omega_{0}^{2}=\xi / m .
$$

In Eq. (7) we see two causes of dispersion. Namely, the first constituent under the radical appears because of a discrete nature of the model, while the second one is due to the presence of a cutoff frequency $\Omega$, leading to a forbidden band in the frequency spectrum.

We will focus mainly on the second type of dispersion that comes from the cutoff frequency and allows us to introduce a simpler continuous version of the problem, making the transition to the limit $a \rightarrow 0$. Thus, replacing the mass number by the coordinate $x=n a$, and the energy of the primitive cell by the density of energy $\mathcal{E}(x)=\mathcal{E}_{n} / a$, introducing new constants

$$
\rho=m / a, G=\kappa / a, T=\xi a, c=a \omega_{0}=\sqrt{T / \rho},
$$

and variables

$$
\begin{aligned}
& t \rightarrow \Omega^{-1} t, \quad x \rightarrow c \Omega^{-1} x, \\
& \mathcal{E} \rightarrow \rho \Omega^{2} \mathcal{E}, \quad J \rightarrow c \rho \Omega^{2} J,
\end{aligned}
$$

we replace Eqs. (2b), (4) and (5) by the following more simple and convenient system of equations:

$$
\begin{aligned}
& u_{t t}=u_{x x}-u, \\
& \mathcal{E}(x)=\frac{1}{2}\left(u_{t}^{2}+u^{2}+u_{x}^{2}\right), \\
& J(x)=-u_{t} u_{x} .
\end{aligned}
$$

In fact, the dispersion relation of Eq. (11a)

$$
\omega=\sqrt{k^{2}+1}
$$

is the long wave approximation $(k a \ll 1)$ of Eq. (7). The set (11) of dimensionless equations will be our main tool. Equation (11a) is the well-known one-dimensional Klein-Gordon equation, and it also describes the waves in an elastically braced string [10]. It was discussed in detail in Matulis's paper [11].

\section{Energy flow in an individual wave}

First, we consider the energy flow in the case of an individual wave, running to the right along the $x$ axis.

We present its amplitude in the real form

$$
u(x, t)=\cos \varphi, \quad \varphi=k x-\omega t
$$

because the energy and its flow that interest us are the square functions of the wave amplitude, and thus, the complex exponent form is not convenient. The amplitude (13) satisfies Eq. (11a) automatically if the frequency $\omega$ and the wave number $k$ are related by Eq. (12).

Substituting the amplitude (13) into Eqs. (11b, c), we find expressions of energy

$$
\begin{aligned}
& \mathcal{E}=\frac{1}{2}\left[\left(\omega^{2}+k^{2}\right) \sin ^{2} \varphi+\cos ^{2} \varphi\right] \\
& =\frac{\omega^{2}}{2}-\frac{k^{2}}{2} \cos (2 \varphi),
\end{aligned}
$$

and its flow

$$
J=\omega k \sin ^{2} \varphi
$$

When interested in mean values, the averages of energy density and flow over the period of rapidly changing phase $\varphi$

$$
\langle E\rangle=\omega^{2} / 2, \quad\langle J\rangle=\frac{k \omega^{2}}{2 \omega}=v_{\mathrm{gr}}\langle E\rangle
$$

are used. Here the symbol

$$
v_{\mathrm{gr}}=\mathrm{d} \omega / \mathrm{d} k=k / \omega
$$

stands for the group velocity. 
We see that although the mean values show a fairly monotonous picture (the uniform wave energy is transferred with a group velocity), the individual wave demonstrates the oscillating flow of energy, and there are points (corresponding to phase values $\varphi_{n}=\pi n$ ) where this flow is zero. Therefore, it is hoped that the superposition of several such waves could show even a negative local flow. In the next section, we will consider the two-wave superposition, which, in the case of Schrodinger's 1D equation, demonstrated the negative flow of probability [2].

\section{Superposition of two waves}

Let us consider the following superposition of two waves

$$
u(x, t)=\cos \varphi_{1}+A \cos \varphi_{2}
$$

where the symbol

$$
\varphi_{\mathrm{i}} \equiv \varphi_{\mathrm{i}}(x, t)=k_{i} x-\omega_{i} t
$$

stands for the individual wave phase. According to Eq. (11c) it corresponds to the following flow of energy (for the sake of convenience divided by $4 \omega_{1} k_{1} / \pi^{2}$ )

$$
\begin{aligned}
J_{0}=\frac{\pi^{2} J}{4 \omega_{1} k_{1}}= & \frac{\pi^{2}}{4}\left(\sin \varphi_{1}+f \sin \varphi_{2}\right) \\
& \times\left(\sin \varphi_{1}+g \sin \varphi_{2}\right),
\end{aligned}
$$

where

$$
f=\frac{\omega_{2}}{\omega_{1}} A, \quad g=\frac{k_{2}}{k_{1}} A=\frac{v_{\mathrm{gr}, 2}}{v_{\mathrm{gr}, 1}} f .
$$

We see that the dispersion is really important, because in the case of its absence the group velocities are equal $\left(v_{\mathrm{gr}, 1}=v_{\mathrm{gr}, 2}\right)$. Consequently, $f=g$, the expression on the right-hand side of Eq. (20) is a function squared, and the energy flow cannot be negative.

Our goal is to identify the $f$ and $g$ values in which there is a backflow in the widest interval for phases and the definition of the limits of this flow itself. Let us keep in mind that because of the periodicity of flow (20) it is enough to consider it in the finite square area $0 \leqslant \varphi_{1}, \varphi_{2}<2 \pi$. In addition, the backflow is possible only in the case when the parentheses in Eq. (20) are of different signs.
Obviously, this can only happen when trigonometry functions $\sin \varphi_{1}$ and $\sin \varphi_{2}$ also have different signs. This reduces the search area to two smaller squares. We choose one of them $0<\varphi_{1}<\pi$, $\pi<\varphi_{2}<2 \pi$, because the another turns out to be a simple replacement of variables $\varphi_{i} \rightarrow 2 \pi-\varphi_{i}$ and leads to a similar result. For convenience, we will change one of the phases: $\varphi_{2} \rightarrow 2 \pi-\varphi_{2}$. Then the flow which we are interested in is defined in the $0 \leqslant \varphi_{1}, \varphi_{2}<\pi$ square, and reads

$$
J_{0}=\frac{\pi^{2}}{4}\left(\sin \varphi_{1}-f \sin \varphi_{2}\right)\left(\sin \varphi_{1}-g \sin \varphi_{2}\right) .
$$

This expression is symmetrical to the replacement of variables: $\varphi_{i} \rightarrow \pi-\varphi_{i}$. Therefore, the search area can be narrowed down to the next square:

$$
0 \leqslant \varphi_{1}, \varphi_{2}<\pi / 2 .
$$

Note that the sinus functions in the above interval are the monotonously growing ones. So it is convenient to replace them with simple variables as follows:

$$
\sin \varphi_{1}=2 \xi / \pi, \quad \sin \varphi_{2}=2 \eta / \pi .
$$

This allows us to replace the flow of energy (22) with the following polynomial

$$
J_{0}=(\xi-f \eta)(\xi-g \eta)=F \cdot G
$$

which we have to consider in the $0 \leqslant \xi, \eta \leqslant 1$ square.

Now it is very easy to set the boundaries of the negative flow area: one only needs to find the lines where this flow is zero. Since Eq. (25) consists of a product of two factors, it is zeroed when any of them is zero. In this way, we define two oblique straights, shown in Fig. 2 by (red online) solid and (blue online) dashed lines. Each of them separates the area where the corresponding factor ( $F$ or $G$ ) is positive from the area where it is negative. These two straights together with the edges of the square define the area shown in yellow, where the backflow exists. From the equation

$$
F=\xi-f \eta=0
$$

and Fig.2, it is obvious that the smaller the coefficient $f$, the higher the straight. So to have the maximum backflow area, we need to make as much difference 


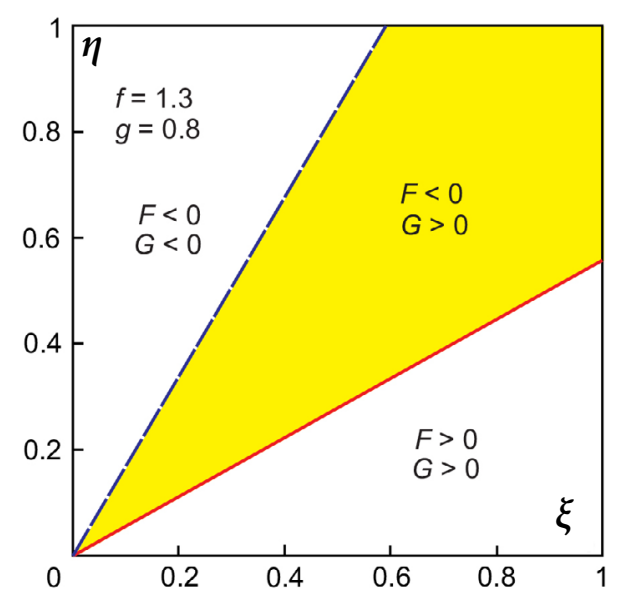

Fig. 2. The area of the negative energy flow.

as possible between the coefficient $f$ and $g$ values. For instance, we can choose

$$
f \rightarrow \infty, \quad g=0 .
$$

In this case the flow becomes $J_{0} \sim-f \xi \eta$, and it is negative on the whole square of interest. However, according to the definitions (21) in order to realize this favourable situation, we need the fulfilment of the following inequalities

$$
\omega_{2}>\omega_{1}, \quad k_{2}<k_{1} \text {, }
$$

that is actually the definition of meta-material [12]. This points to the close relationship of the local backflow of energy under consideration with the popular area of the design and research of meta-materials, for which this backflow is the main property.

Restricting our consideration with the conventional models, we will take into account the dispersion relation given by Eq. (12). In this case, the ability to have a local backflow can be evaluated as follows. Since the dispersion for the backflow problem is essential, it makes sense to choose the wave vectors from the interval $\{0,1\}$ where this dispersion is the largest. So taking into account Eq. (12) let us choose

$$
k_{1} \rightarrow 0, \quad \omega_{1}=1, \quad k_{2}=1, \quad \omega_{2}=\sqrt{2} .
$$

Then the parameters and flow normalized by the largest positive value will be as follows:

$$
\begin{aligned}
& f=\omega_{2} / \omega_{1}=\sqrt{2}, \quad g \rightarrow \infty, \\
& J_{0}=\eta(\eta-\xi / \sqrt{2}) .
\end{aligned}
$$

This flow is shown in Fig. 3. The zero plane is highlighted by a dashed (yellow online) rectangle, and the (red online) straights indicate the sector of

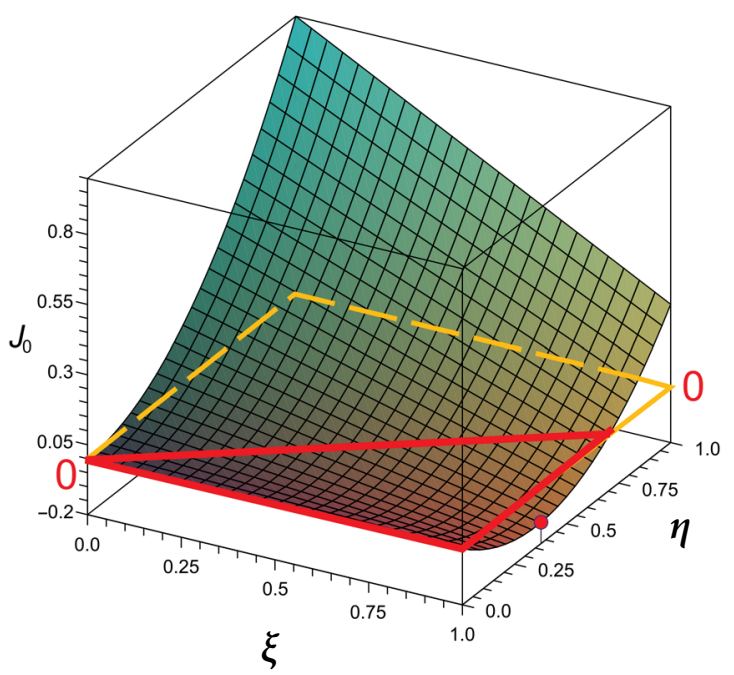

Fig. 3. Energy flow in the case of dispersion relation (12).

backflow. We see that the maximum backflow value is reached on the right side of the square (indicated by a small red circle) at the point $\xi_{0}=1, \eta_{0}=1 / 2 \sqrt{2}$. This value reads

$$
J_{0, \max }=-0.125 \text {, }
$$

that is about an order of magnitude more than similar values received for a free electron in a quantum description [2].

Considering wave processes, scientists are often interested not only in the instant values of wave characteristics but also in their means: the values averaged by a period. In the case of the superposition of two waves, it makes sense to consider the waves of close frequencies. In this case, it can be expected that these characteristics, averaged over the fast process, still contain useful information about slow beats in both temporal and coordinate dependences. To illustrate this possibility, let us look at the superposition of two waves with close to one frequencies $\left(\omega_{1}, \omega_{2} \sim 1\right)$, and

$$
k_{1}=0, \quad k_{2} \equiv k \ll 1
$$

We write then

$$
u(x, t)=A \cos t+\cos (k x-t)
$$


The energy flow of this wave is

$$
J=-u_{x} u_{t}=k \sin (k x-t)[-A \sin t+\sin (k x-t)]
$$

Now, with the average of trigonometric functions in mind

$$
\left\langle\sin ^{2} t\right\rangle=\left\langle\cos ^{2} t\right\rangle=1 / 2, \quad\langle\sin t \cos t\rangle=0,
$$

we obtain the following expression of the averaged flow:

$$
\langle J\rangle=(k / 2)[1+A \cos (k x)] .
$$

We see that by adding to the running wave some homogeneous oscillations of amplitude $A$, we obtain a modulation of the flow of energy by the same amplitude $A$. When the amplitude exceeds unity $(A>1)$, then the local backflow appears.

\section{Green's function}

The possibility of a backflow in the case of two cosine waves, discussed in the previous section, suggests the study of more complex superpositions, including packages. That is why we will consider the limiting case of short excitation, namely, the reaction of the string to the instant point impact, which is described by the equation

$$
G_{t t}-G_{x x}+G=\delta(x) \delta(t)
$$

and boundary conditions

$$
\begin{aligned}
& G( \pm \infty, t)=0, \\
& \left.G(x, t)\right|_{t<0}=0, \\
& \left.G_{t}(x, t)\right|_{t<0}=0 .
\end{aligned}
$$

Actually it is the equation for Green's function of our basic Eq. (11a).

A successful solution of this equation can be obtained by analytical means. Indeed, first we will replace this equation with a homogeneous one, replacing the Dirac functions by the proper initial condition:

$$
\begin{aligned}
& G_{t t}-G_{x x}+G=0, \\
& G( \pm \infty, t)=0,
\end{aligned}
$$

$$
G(x, 0)=0,
$$

$$
G_{t}(x, 0)=\delta(x) .
$$

Next, we present the solution in the form of a Fourier transform

$$
G(x, t)=\frac{1}{2 \pi} \int_{-\infty}^{\infty} \mathrm{d} k \mathrm{e}^{\mathrm{i} k x} g(k, t) .
$$

Now, by substituting this expression into Eqs. (39) and using the Fourier transform of Dirac function

$$
\delta(x)=\frac{1}{2 \pi} \int_{-\infty}^{\infty} \mathrm{d} k \mathrm{e}^{\mathrm{i} k x},
$$

we convert the Green's function equations into the following ordinary differential equation:

$$
\begin{aligned}
& \frac{\mathrm{d}^{2}}{\mathrm{~d} t^{2}} g(k, t)+\left(1+k^{2}\right) g(k, t)=0, \\
& g(k, 0)=0, \\
& \left.\frac{\mathrm{d}}{\mathrm{d} t} g(k, t)\right|_{t=0}=1 .
\end{aligned}
$$

It is easy to see that the solution of Eq. (42a), satisfying the initial conditions $(42 \mathrm{~b}, \mathrm{c})$, reads

$$
g(k, t)=\frac{\sin \left(t \sqrt{1+k^{2}}\right)}{\sqrt{1+k^{2}}} .
$$

Substituting it into Eq. (40) we obtain the Green's function

$$
G(x, t)=\frac{1}{2 \pi} \int_{-\infty}^{\infty} \mathrm{d} k \mathrm{e}^{\mathrm{i} k x} \frac{\sin \left(t \sqrt{1+k^{2}}\right)}{\sqrt{1+k^{2}}} .
$$

Using the known representation of the exponential function

$$
\mathrm{e}^{\mathrm{i} k x}=\cos (k x)+\mathrm{i} \sin (k x)
$$

and bearing in mind that only the even part of the function contributes to integral (44), it is possible to simplify further this expression by converting the integrand into the real function:

$$
G(x, t)=\frac{1}{\pi} \int_{0}^{\infty} \mathrm{d} k \cos (k x) \frac{\sin \left(t \sqrt{1+k^{2}}\right)}{\sqrt{1+k^{2}}} .
$$

Such integral can be found in the reference book [13], and the Green's function can be presented in the final form 


$$
G(x, t)=\frac{1}{2} J_{0}\left(\sqrt{t^{2}-x^{2}}\right) \Theta(t-x),
$$

where the symbol $J_{0}(x)$ stands for the Bessel function of the zero order, and the symbol $\Theta(x)$ is for the Heaviside step function. It is shown by a dashed (blue online) curve in Fig. 4. We see that after hitting the string at the initial moment of time $(t=0)$, a short pulse (precursor) runs away with constant velocity, pulling behind itself slowly decaying waves.

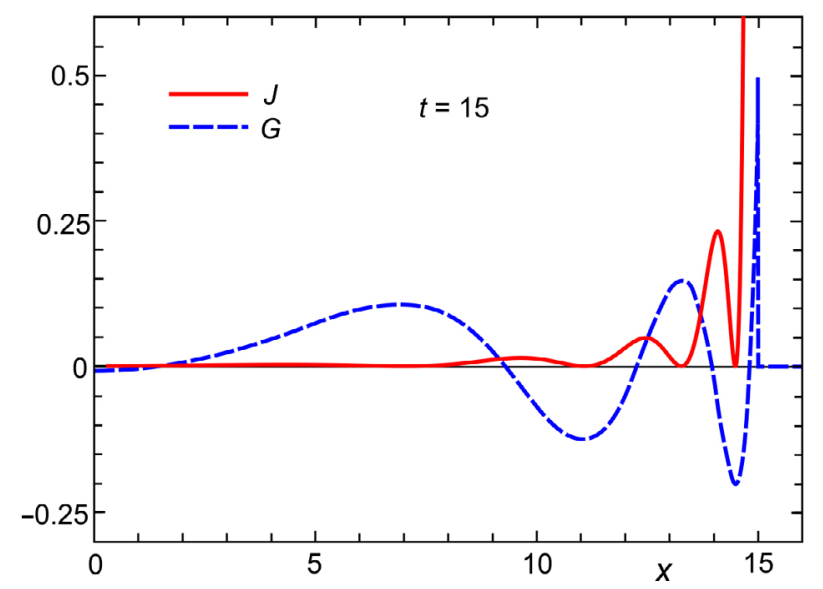

Fig. 4. Green's function (a dashed (blue online) curve) and the corresponding energy flow (a solid (red online) curve).

According to Eq. (11c) we define the energy flow corresponding to the obtained Green's function:

$$
J(x, t)=-G_{t}(x, t) G_{x}(x, t)=\frac{x t J_{1}^{2}\left(\sqrt{t^{2}-x^{2}}\right)}{4\left(t^{2}-x^{2}\right)} .
$$

It is shown by a solid (red online) curve in the same Fig. 4. Note that the flow of energy is similar to that one seen in the case of an individual cosine wave: it has zeros at certain wave phases, corresponding to the extremes of the wave amplitude. Therefore, it should be expected that in the case of two successive strikes, the propagating excitation will possibly demonstrate a local backflow. In fact, it is so, as demonstrated by Fig. 5. The areas of the $x t$ plane, where the backflow is observed, are painted (red online).

Comparing the results obtained in the last two sections, it may be concluded that a local backflow of energy is possible when various excitations interfere. The backflow manifests itself in the greatest way when two cosine waves interfere with each other.

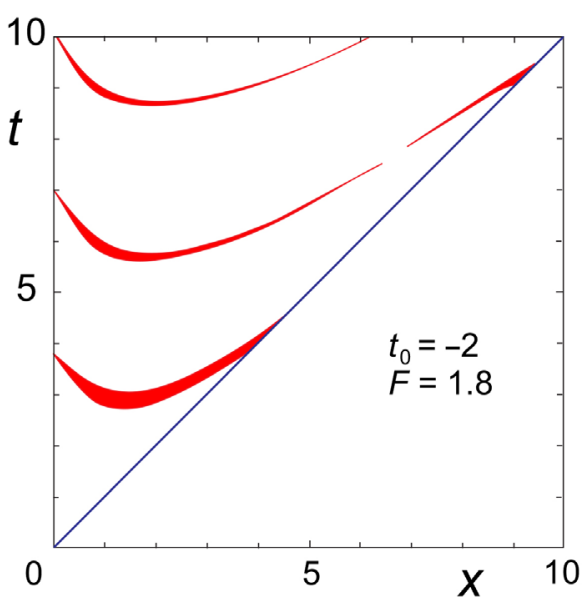

Fig. 5. Contour plot of energy flow in the string exited by two successive strikes, namely, when $\delta(t)$ in Eq. (37) is replaced by $\delta(t)+F \delta\left(t+t_{0}\right)$.

\section{Harmonic oscillator}

The wave systems considered in the previous sections were infinite. Trying to detect the backflow experimentally, however, one has to deal with the finite system, in which the reflection of a wave from its edges either suppresses the flow of energy, or distorts it significantly. Therefore, considering the finite systems one has to modify the problem, including both the wave generation at one edge of the chain and the wave absorption on the other one, for instance, including the dissipating force (say, friction) into the equation for the last mass in the chain shown in Fig. 1.

Such modification does not complicate the consideration significantly and actually leaves the result valid concerning the backflow if the dissipation is small enough, almost regardless of the length of $1 \mathrm{D}$ lattice. In support of the words said in this section, we will look at an ultrashort chain consisting only of one mass, which is both the beginning and the end of this chain. Therefore, this mass is simultaneously under the influence of the external force and the dissipating one as well. This simple system is shown in Fig. 6. In fact, it is a harmonic oscillator, the coordinate of which satisfies the following equation:

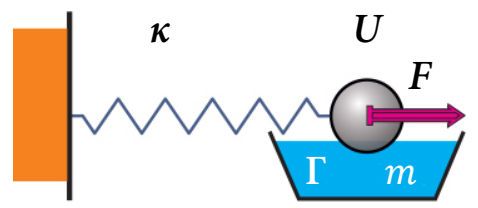

Fig. 6. Harmonic oscillator. 


$$
m \ddot{u}+\Gamma \dot{u}+\kappa u=F(t) .
$$

Here the symbol $F(t)$ stands for the external force, and the dissipation is characterized by the friction coefficient $\Gamma$. Presenting this well-known problem, we aim to illustrate the assertion stated in the Introduction that the backflow of energy can appear in any oscillating system if the energy flow can be split into several channels.

For the sake of convenience let us again introduce the dimensionless variables

$$
\begin{aligned}
& t \rightarrow \Omega^{-1} t, \quad \Omega=\sqrt{\kappa / m}, \quad \gamma=\Gamma / m \Omega, \\
& u \rightarrow u F / m \Omega^{2},
\end{aligned}
$$

(here, the symbol $F$ stands for the characteristic amplitude of external force, namely $F(t)=F f(t)$ ) and rewrite Eq. (49) as follows:

$$
\ddot{u}+\gamma \dot{u}+u=f(t) .
$$

Now following the ideas of Section 3, we multiply Eq. (51) by $\dot{u}$ and get the standard energy conservation law

$$
\frac{\mathrm{d}}{\mathrm{d} t} \mathcal{E}=\frac{\mathrm{d}}{\mathrm{d} t}\left(\frac{1}{2} \dot{x}^{2}+\frac{1}{2} x^{2}\right)=\dot{x} f-\gamma \dot{x}^{2},
$$

which shows that the total energy of the oscillator $\mathcal{E}$ changes due to the incoming flow of energy

$$
J=\dot{u} f
$$

from the external force and the leaking flow

$$
D=\gamma \dot{u}^{2},
$$

flowing to the thermostat (see the water vessel in Fig. 6). Both of these quantities indicate the main flow of energy through mass from the pumping force to the thermostat.

Recall that our goal is to consider the system with possible branching of the flow. Exactly this type of branching appears in the oscillator in question. Indeed, if we write down separately the balance for the kinetic energy of the mass and the potential energy of the spring

$$
\frac{\mathrm{d}}{\mathrm{d} t} T=\frac{\mathrm{d}}{\mathrm{d} t}\left(\frac{1}{2} \dot{u}^{2}\right)=\dot{u} \ddot{u}=\dot{u} f-u \dot{u}-\gamma \dot{u}^{2},
$$

$$
\frac{\mathrm{d}}{\mathrm{d} t} V=\frac{\mathrm{d}}{\mathrm{d} t}\left(\frac{1}{2} u^{2}\right)=u \dot{u},
$$

we see that in addition to the above-mentioned direct flow of energy, there is an additional flow

$$
W=u \dot{u},
$$

taking off the energy from the main flow to the spring. Let us check if there is a possibility of the negative flow $J<0$ of energy, which could be called an analog to the previously considered backflow in the infinite chain.

First, we consider the case when the force is of a single cosine type component:

$$
f(t)=\cos (\omega t)=\operatorname{Re} \mathrm{e}^{-\mathrm{i} \omega t} .
$$

The standard stationary solution of Eq. (51) reads

$$
\begin{aligned}
u & =\operatorname{Re} \frac{\mathrm{e}^{-\mathrm{i} \omega t}}{1-\omega^{2}-\mathrm{i} \gamma \omega} \\
& =\frac{\left(1-\omega^{2}\right) \cos (\omega t)+\gamma \omega \sin (\omega t)}{\left(\omega^{2}-1\right)^{2}+\gamma^{2} \omega^{2}}, \\
\dot{u} & =a(\omega) \cos (\omega t)+b(\omega) \sin (\omega t),
\end{aligned}
$$

where the amplitudes

$$
\begin{aligned}
& a(\omega)=\frac{\gamma \omega^{2}}{\left(\omega^{2}-1\right)^{2}+\gamma^{2} \omega^{2}}, \\
& b(\omega)=\frac{\omega\left(\omega^{2}-1\right)}{\left(\omega^{2}-1\right)^{2}+\gamma^{2} \omega^{2}},
\end{aligned}
$$

actually are the real and imaginary parts of Lorenzian.

Using the obtained expressions for the coordinate of the oscillator and its velocity, we determine the energy flows that are of interest to us:

$$
\begin{aligned}
& J=\frac{a(\omega)}{2}+\frac{\omega \sin \left(2 \omega t+\varphi_{J}\right)}{2 \sqrt{\left(\omega^{2}-1\right)^{2}+\gamma^{2} \omega^{2}}}, \\
& D=\frac{a(\omega)}{2}\left[1+\sin \left(2 \omega t+\varphi_{D}\right)\right], \\
& W=\frac{\omega \cos \left(2 \omega t+\varphi_{D}\right)}{2\left[\left(\omega^{2}-1\right)^{2}+\gamma^{2} \omega^{2}\right]}
\end{aligned}
$$

where 


$$
\begin{aligned}
& \varphi_{J}=\arctan [a(\omega) / b(\omega)], \\
& \varphi_{D}=\arctan \left[\frac{\gamma^{2} \omega^{2}-\left(\omega^{2}-1\right)^{2}}{2 \gamma \omega\left(\omega^{2}-1\right)}\right] .
\end{aligned}
$$

These flows together with kinetic $T$ and potential $V$ energies are shown in Fig. 7 .

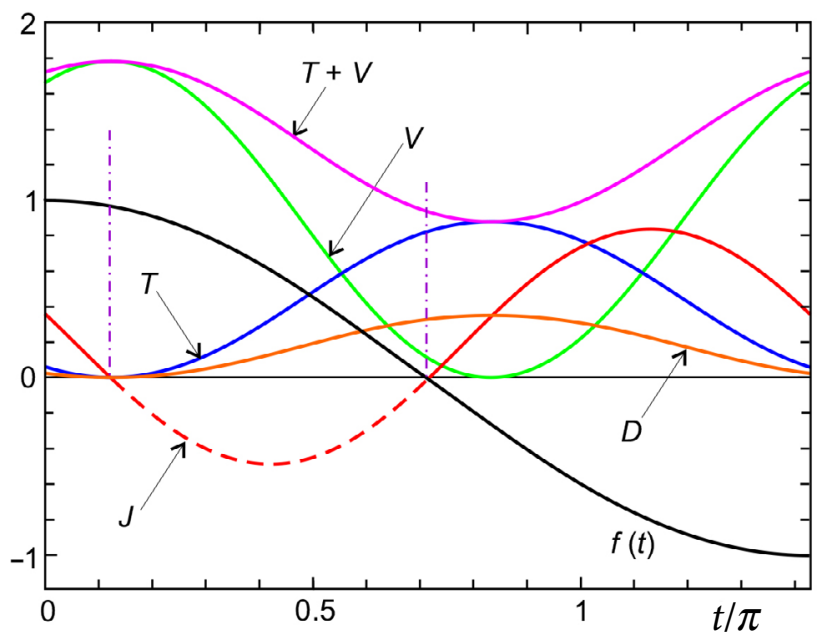

Fig. 7. Energies and flows given by formulas (52), (55), (57) and (60a), $\omega=0.7, \gamma=0.2$.

Paying attention to the obtained formulas and Fig. 7 , we can note some interesting points. The averaged flows over oscillations with double frequency

$$
\langle J\rangle=\langle D\rangle=a(\omega) / 2
$$

are positive and indicate that the main flow of energy goes from the external force to the thermostat. The magnitude of this flow coincides with the amplitude of the oscillating part of the dissipative flow $D$ (see the second term in the right-hand side of Eq. $(60 \mathrm{~b}))$. This confirms that this flow is always positive, which it should be, because the dissipative part of the system has no source of energy. Meanwhile, the pumping energy (flow $J$ ) can take on negative values, because the amplitude of the second term in the right part of Eq. (60b) is always larger than its first term, except for the point of resonance $(\omega= \pm 1)$, where both terms are equal. This negative part of the pumping energy is highlighted by the dashed part of the $J$ curve. This is a rather trivial property of the forced oscillator, which is often overlooked, because most often only the average power absorption is of interest. In our case, as mentioned above (see Eq. (62)), it is always positive.
To get a negative average pumping power that manifests itself over a long period of time, one needs to try to excite the oscillator with a force consisting of two periodic functions of close frequency, for instance, postulating the external force as follows:

$$
\begin{aligned}
f(t) & =\cos [(\omega-\Delta \omega / 2) t]+\cos [(\omega+\Delta \omega / 2) t] \\
& \equiv f_{-}+f_{+}, \quad \Delta \omega \ll \omega .
\end{aligned}
$$

Let us note that such a double excitation of the oscillator is a good analog to the previously considered problem of two cosine wave propagation in an infinite chain. Here and in further terms for brevity we will use \pm indexing of functions instead of indicating explicitly their dependence on shifted frequencies $\omega_{+}=\omega \pm \Delta \omega / 2$. Therefore, according to Eq. (53), the energy pumped into the oscillator by the exciting force can be represented in the form

$$
J=\dot{u}_{-} f_{-}+\dot{u}_{+} f_{+}+\dot{u}_{-} f_{+}+\dot{u}_{+} f_{-}
$$

where the expressions for velocities $\dot{u}_{ \pm}$follow from Eq. (58b) by proper replacement of frequency.

To calculate the average of this equation, one needs the following averages of trigonometry function products

$$
\begin{aligned}
& \left\langle\cos ^{2}[(\omega \pm \Delta \omega / 2) t]\right\rangle=1 / 2, \\
& \langle\cos [(\omega \pm \Delta \omega / 2) t] \sin [(\omega \pm \Delta \omega / 2) t]\rangle=0, \\
& \langle\cos [(\omega+\Delta \omega / 2) t] \cos [(\omega-\Delta \omega / 2) t]\rangle \\
& =\langle[\cos (\omega t) \cos (\Delta \omega t / 2)-\sin (\omega t) \sin (\Delta \omega t / 2)] \\
& \times[\cos (\omega t) \cos (\Delta \omega t / 2)+\sin (\omega t) \sin (\Delta \omega t / 2)]\rangle \\
& =\left[\cos { }^{2}(\Delta \omega t / 2)-\sin ^{2}(\Delta \omega t / 2) / 2=\frac{1}{2} \cos (\Delta \omega),\right. \\
& \langle\cos [(\omega \pm \Delta \omega / 2) t] \sin (\omega \mp \Delta \omega / 2) t]\rangle \\
& =\mp \frac{1}{2} \sin (\Delta \omega),
\end{aligned}
$$

which can be calculated assuming that the averaging over fast oscillations with frequency $\omega$ does not affect the slow beats. 
The averaging of two first terms in Eq. (64) is simple: it leads to

$$
\begin{aligned}
& \left\langle\dot{u}_{-} f_{-}\right\rangle+\left\langle\dot{u}_{+} f_{+}\right\rangle=\left(a_{-}+a_{+}\right) / 2 \\
& \equiv[a(\omega-\Delta \omega / 2)+a(\omega+\Delta \omega / 2)] / 2=A(\omega),
\end{aligned}
$$

that gives only a permanent background. Meanwhile, the averaged two remaining terms demonstrate the beatings:

$$
\begin{aligned}
& \left\langle\dot{u}_{-} f_{+}\right\rangle=\left[a_{-} \cos (\Delta \omega t)-b_{-} \sin (\Delta \omega t)\right] / 2, \\
& \left\langle\dot{u}_{+} f_{-}\right\rangle=\left[a_{+} \cos (\Delta \omega t)+b_{+} \sin (\Delta \omega t)\right] / 2 .
\end{aligned}
$$

Putting all four of the above terms together, we get the final expression of the pumping power

$$
\langle J\rangle=A(\omega)[1+\cos (\Delta \omega t)]+B(\omega) \sin (\Delta \omega t)
$$

where

$$
B(\omega)=\left(b_{+}-b_{-}\right) / 2 .
$$

It is remarkable that averaging Eq. (68) over the period of beats $(2 \pi / \Delta \omega)$ we obtain $\langle J\rangle=A$, that coincides with the average dissipation flow. Another good fact for us is that the amplitude of the oscillating part of Eq. (68) is equal to $\sqrt{A^{2}+B^{2}}$, and it is always larger than its constant part $A$. Thus, there is always a certain phase interval where the mean pumping flow is negative, i.e. there is an analog to backflow which we are looking for. This backflow is larger and appears in a wider phase interval, the greater the difference $(B-A)$. To get an idea when this is possible, let us take a look at

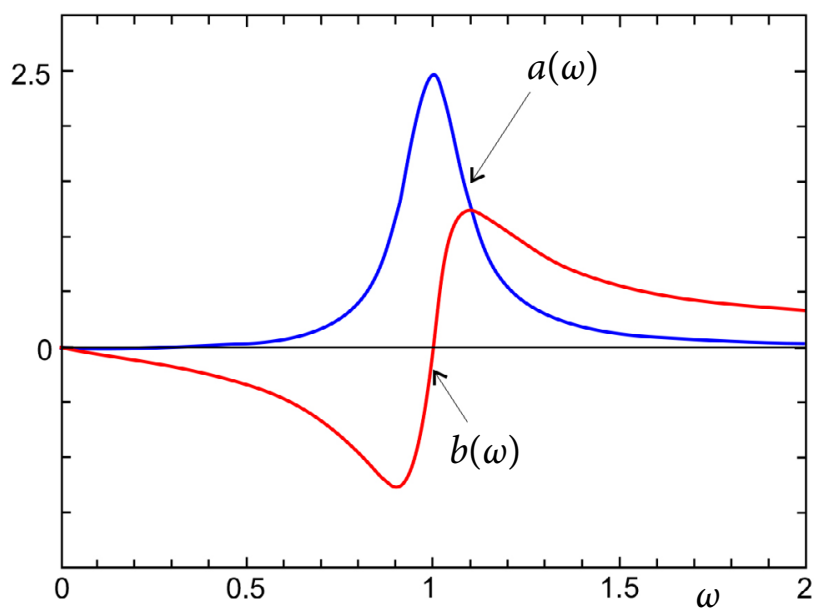

Fig. 8. Auxiliary functions (59).
Fig. 8, where the frequency dependences of auxiliary functions (59) are shown. Because the function $B$ consists of a difference of two functions $b$ at close frequencies (it can be seen as a derivative of function $B$ by frequency), its value is the largest in the resonance region $(\omega \approx 1)$, as seen in Fig. 8. We can see this in Fig. 9, where the functions $A$ and $B$ are shown in the case of a concrete detuning. We see that the function $B$ has the largest value (even exceeding the value of function $A$ ) at the resonance point $(\omega=1)$.

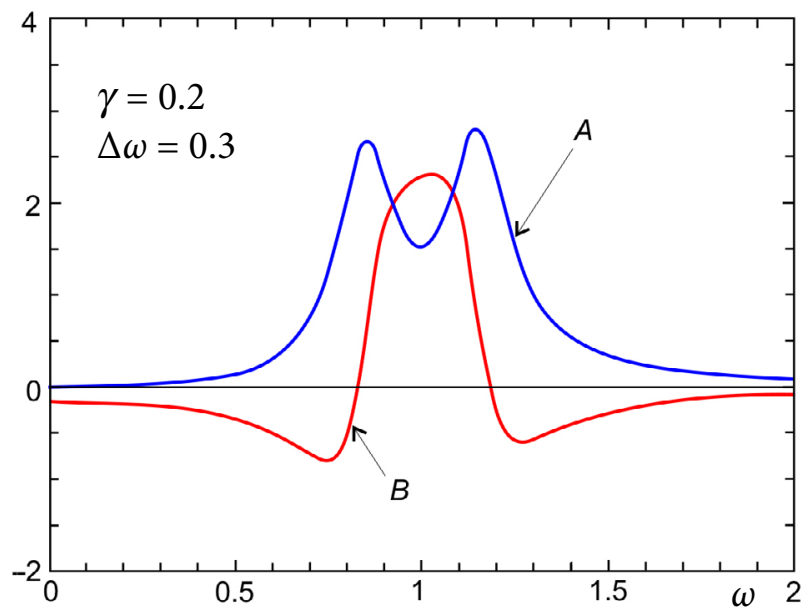

Fig. 9. Functions $A$ and $B$.

Figure 10 sums up this simple reasoning. Here the temporal dependences of the above-mentioned functions are shown. In part (a) of this graph, the external force (63) is shown by a black dashed curve, and a (blue online) solid curve depicts the velocity $\dot{u}$ of the oscillator, calculated according to Eq. (58b). Both of these curves clearly demonstrate the beats, that should be the case due to the proximity of the frequencies of two cosine type components of the external force, and the lag in the velocity phase.

In part (b) of the figure, we see the corresponding energy flows caused by driving force: a (blue online) dashed curve shows the instantaneous value of the flow $J$, and a solid (red online) one is its averaged value according to Eq. (68).

We see that the instantaneous flow $J$ has negative values at some interval of time of each period of the mean frequency, as was the case with a single-component external force. The averaged flow also exhibits negative values (see the dashed part of the (red online) curve), that take up a significant portion of the beating period. 

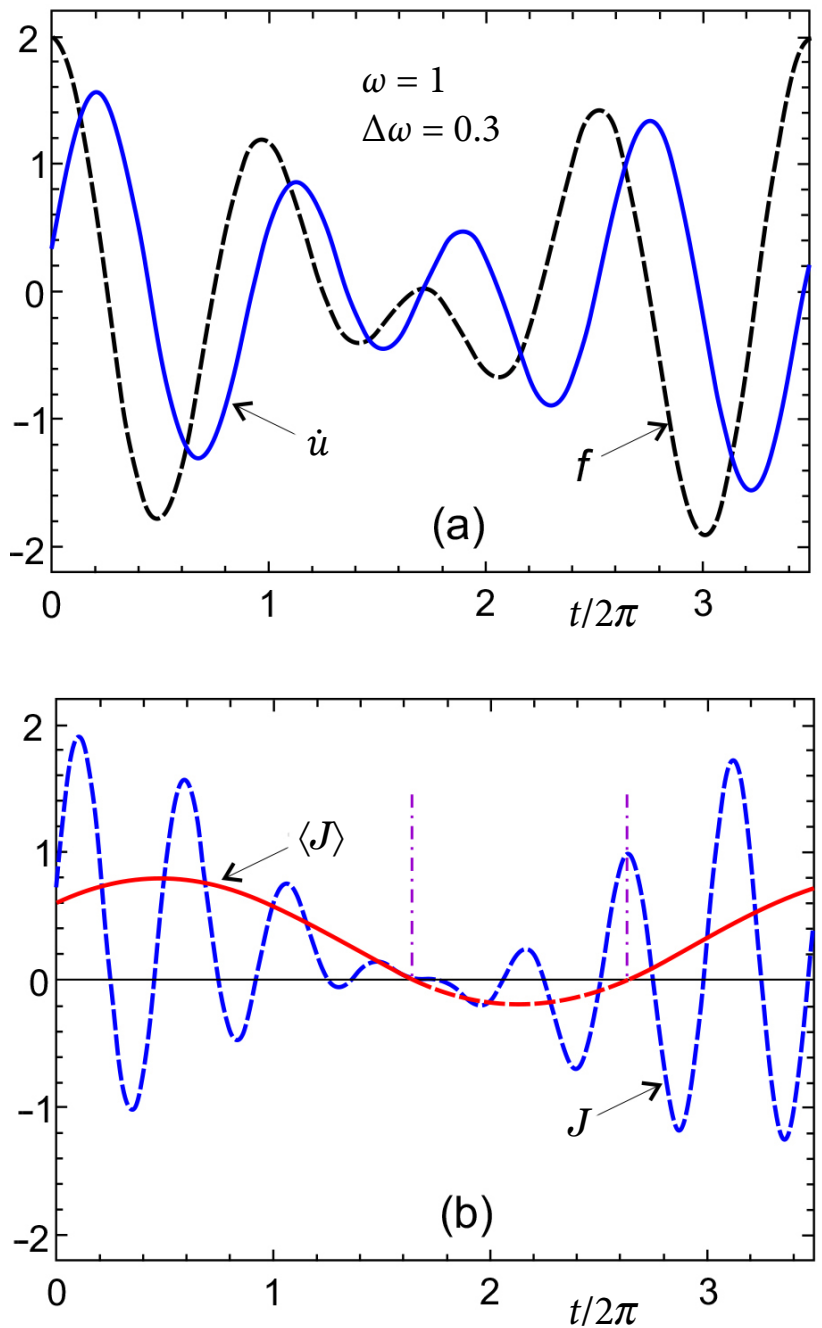

Fig. 10. Oscillator excited by the external force consisting of two periodic components: (a) temporal behaviour of force and velocity, (b) pumping energy and its averaged value.

\section{Conclusions}

We have shown that in the classical system described by differential equations with a spectrum that has a cutoff frequency, there is the possibility of a backflow of energy localized both by coordinate and time as in the known in literature case of quantum mechanics. The main condition for the appearance of this backflow is the wave dispersion, which appears due to the presence of local modes in the medium of propagation. The most favourable opportunity occurs when two waves of slightly different frequencies are propagating. In this case, the backflow occurs both in the microscopic description and using averages.

We illustrated these claims by considering the simplest mechanical system consisting of a chain of masses connected by springs between the nearest neighbours and by additional springs attached to stationary supports, that actually ensures the appearance of the local modes.

Concerning propagation of packages, we studied Green's function for the above-mentioned system and showed that the conditions for backflow are not so favourable. However, this is also possible in the case with two consecutive well-coordinated strikes.

We claim that the backflow appears as a result of the branching of energy flows, and demonstrate this on the example of the simplest model of a forced damped oscillator. Here the backflow (the return of energy to external power) is quite impressive. The instantaneous backflow is already observed at the excitation by a single cosine type force component. In the case of two cosine type force components of close frequency the averaged backflow takes place.

The proposed mechanical chain can be realized in an experimentally simple way. The characteristic frequencies in the $\mathrm{Hz}$ range are easy to observe in a complete analogy with the Süsstrunk and Huber experiments [14], where the pendulum system was used to demonstrate edge modes in quantum mechanics.

It is also worth noting that from a mathematical point of view the mechanical system considered is equivalent to an electric one, namely, a transmission line made up of cells shown in Fig. 11 . Using Kirchhoff's equations, it is easy to show that the currents $I_{n}$ of inductancies $L$ are described by the same type Eq. (2b) as coordinates of the masses of the considered chain. We believe that using such a transmission line it is possible to observe the considered phenomena of energy backflow in the $\mathrm{MHz}$ range.

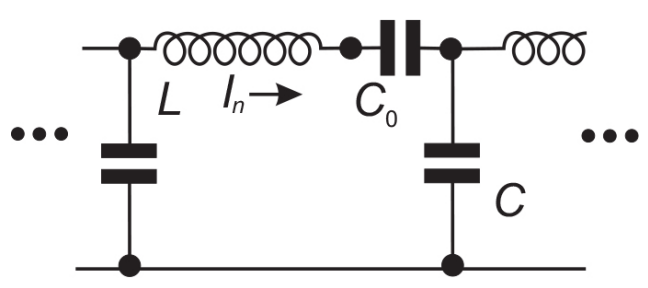

Fig. 11. Cell of the transmission line.

\section{References}

[1] G.R. Allcock, The time of arrival in quantum mechanics III. The measurement ensemble, Ann. Phys. 53, 311-348 (1969). 
[2] A.J. Bracken and G.F. Melloy, Probability backflow and a new dimensionless quantum number, J. Phys. A 27, 2197-2211 (1994).

[3] J.M. Yearsley, J.J. Halliwell, R. Hartshorn, and A. Whitby, Analytical examples, measurement models, and classical limit of quantum backflow, Phys. Rev. A 86, 042116-1-13 (2012).

[4] A. Goussev, Probability backflow for correlated quantum states, Phys. Rev. Res. 2, 033206 (2020).

[5] A. Goussev, Quantum backow in a ring, arXiv: quant-ph/2008.08022v1

[6] M.V. Berry, Quantum backflow, negative kinetic energy, and optical retro-propagation, J. Phys. A 43, 415302 (2010).

[7] A.A.D. Villanueva, The negative flow of probability, Am. J. Phys. 88, 325-333 (2020).

[8] A. Goussev, Comment on 'The negative flow of probability', Am. J. Phys. 88, 1023 (2020).
[9] A. Matulis and A. Acus, Classical analog to the Airy wave packet, Lith. J. Phys. 59, 121-129 (2019).

[10]Ph.M. Morse and H. Feshbach, Methods of Theoretical Physics, Vol. 1, Sec. 2.1 (McGraw-Hill Book Company, Inc., New York, 1953).

[11]A. Matulis, Decay of a quasi-stationary state, Lith. J. Phys. 39, 435 (1999).

[12]V.G. Veselago, Properties of materials having simultaneously negative values of the dielectric and magnetic susceptibilities, Usp. Fiz. Nauk 92, 517-526 (1964).

[13]I.S. Gradshteyn and I.M. Ryzhik, Table of Integrals, Series, and Products (Elsevier Academic Press, Amsterdam, New York, 2007) p. 482, 3.8761.

[14]R. Süsstrunk and S.D. Huber, Observation of phononic helical edge states in a mechanical topological insulator, Science 349, 47 (2015).

\title{
NEIGIAMAS MECHANINĖS BANGOS ENERGIJOS SRAUTAS
}

\author{
A. Matulis ${ }^{\mathrm{a}}$, A. Acus ${ }^{\mathrm{b}}$ \\ a Fiziniu ir technologiju mokslu centro Puslaidininkiu fizikos institutas, Vilnius, Lietuva \\ ${ }^{\mathrm{b}}$ Vilniaus universiteto Teorines fizikos ir astronomijos institutas, Vilnius, Lietuva
}

\section{Santrauka}

Aprašoma klasikinè sistema, kuri yra kvantinès sistemos, pasižyminčios neigiamu tikimybès srautu, analogas. Sistemą sudaro tarpusavyje spyruoklemis sujungtų rutuliukų grandinèlè, kurioje kiekvienas rutuliukas papildoma spyruokle dar yra prijungtas prie fiksuotų atramų. Dèl papildomai prijungtų spyruoklių atsiranda plintančių išilgai grandinèlès bangų spektre draustinis dažnių ruožas ir dispersija. Parodyta, kad tai lemia neigiamo bangos energijos srauto atsiradimą. Dviejų interferuojančių bangų atveju šio neigiamo srauto dydis visa eile viršija neigamo tikimybès srauto dydi mi-
\end{abstract}

nètame kvantiniame uždavinyje. Apskaičiuota klasikinę sistemą aprašančių lygčių Gryno funkcija ir parodyta, kad neigiamo energijos srauto atsiradimas imanomas ir tada, kai sistema žadinama dviem trumpais nuosekliais impulsais. Ištirtoje klasikineje sistemoje atsirandantis neigiamo energijos srauto reiškinys aiškinamas energijos išsišakojimu i lokalines modas. Tai patvirtina gauti žadinamo išorine jèga disipacinio osciliatoriaus rezultatai. Parodyta, kad net tokioje paprastoje sistemoje imanomas atbulinis (tiek momentinis, tiek ir vidutinis) energijos srautas. 